\title{
The Conduction Characteristics of Electrical Trees in XLPE Cable Insulation
}

\author{
Ansheng Xie, ${ }^{1,2}$ Xiaoquan Zheng, ${ }^{1}$ Shengtao $\mathrm{Li}^{1}{ }^{1}$ George Chen ${ }^{3}$ \\ ${ }^{1}$ State Key Laboratory of Electrical Insulation and Power Equipment, Xi'an Jiaotong University, \\ Xi'an, Shaanxi 710049, China \\ ${ }^{2}$ School of Environment Science and Engineering, Chang'an University, Xi'an, Shaanxi 710064, China \\ ${ }^{3}$ School of Electronics and Computer Science, University of Southampton, Southampton SO17 1BJ, United Kingdom
}

Received 16 September 2008; accepted 2 June 2009

DOI 10.1002/app.30882

Published online 7 August 2009 in Wiley InterScience (www.interscience.wiley.com).

\begin{abstract}
Depending on the morphology of the material and applied voltage frequency, three kinds of electrical trees can exist in cross-linked polyethylene (XLPE) cable insulation, which are conducting, non-conducting, and mixed trees with different growth mechanisms. It is suggested that when the needle is inserted into large spherulites, conducting trees will form in those spherulites; when it is inserted among spherulites, non-conducting trees will appear along the boundaries of spherulites. Frequency will accelerate the growth of non-conducting trees but have little influence on the initiation and growth processes of conducting trees. If the initiation process of non-conducting trees is too difficult, they will grow into mixed trees.
\end{abstract}

Finally, it is concluded that the space charge limited tiny breakdown around the tips of electrical trees is responsible for the propagation process of conducting trees; on the other hand, fast expansion occurs due to local high temperature and pressure along the boundaries, partial discharge in electrical tree paths and charge recombination, etc., which are the main reason for the growth of nonconducting trees. (C) 2009 Wiley Periodicals, Inc. J Appl Polym Sci 114: 3325-3330, 2009

Key words: cross-linked polyethylene cable insulation; electrical trees; conducting characteristic; morphology; frequency

\section{INTRODUCTION}

Since the first discovery of tree-like aging trace (electrical trees) in organic materials, many articles have been published on the initiation, growth mechanism, and inhibition of electrical trees. However, the research never come to an end for three reasons: First, nowadays, the manufacture level of thick insulation XLPE cable has reached $750 \mathrm{kV}$ rating, and $500 \mathrm{kV}$ rating thick insulation XLPE cable has already been in service. Because of the cooling process during manufacture, the electrical trees are more dangerous in thick cable insulation than low voltage rating cables, which will cause far more loss in power accidents. It is significant to study the initiation and growth mechanisms in XLPE cable insulation so some measures can be taken to avoid or minimize electrical trees in the insulation. Second, the tree-like discharge paths have some similarities with lightning in the air, tree-like discharge in liq-

Correspondence to: A. Xie (xieansheng@gmail.com).

Contract grant sponsor: National Science Funds for Distinguished Young Scholars; contract grant number: 50625721 .

Contract grant sponsor: National Natural Science Foundation of China; contract grant number: 50577052.

Journal of Applied Polymer Science, Vol. 114, 3325-3330 (2009) (C) 2009 Wiley Periodicals, Inc. uid, and tree-like cracking in solid. It has attracted increasing attention in that with the same discharge condition, different discharge paths appear. In recent years, people have spent much effort on simulation of discharge paths in a mathematic way. ${ }^{1-3}$ Third, the development of modern experimental techniques $^{4,5}$ makes it possible to study the characteristics of electrical trees in much detail.

As to the conduction characteristics of electrical trees, Champion and Dodd ${ }^{6}$ established the partial discharge model to simulate the characteristics of conducting and non-conducting electrical trees in epoxy resins and suggested that the accumulation state of epoxy resins will affect the conducting characteristics of electrical trees. Electrically conducting trees were found to form in the glassy state and non-conducting trees formed in the flexible state. In addition, they studied the trees in propylene/ethylene copolymer ${ }^{7}$ and found that in low temperature $\left(128^{\circ} \mathrm{C}\right)$ crystallized copolymer, electrically conducting tree structures were found, while non-conducting tree structures formed in higher temperature $\left(134^{\circ} \mathrm{C}\right)$ crystallized copolymer. However, there are fewer reports on the conducting characteristics of electrical trees in high voltage rating XLPE cable insulation.

In this article, a high voltage generator with wideband frequency, a live microscope digital camera, and special sample preparation process are adopted. 
The initiation, propagation, and structure characteristics of electrical trees generated under different frequencies in semi-crystallization XLPE cable insulation have been investigated; the conducting properties of electrical trees have been decided indirectly according to the color of electrical trees and their growth rates. It is suggested that the electrical trees in XLPE cables insulation can be divided into three types: conducting, non-conducting, and mixed. Finally, the growth mechanisms of conducting and non-conducting trees are explained.

\section{SAMPLE PREPARATION AND EXPERIMENTAL SET-UP}

\section{Sample preparation}

The samples were taken from a $66 \mathrm{kV}$ high voltage XLPE cable supplied by a cable manufacturer. The cable insulation was cut into hollow disc shape with a thickness of $5 \mathrm{~mm}$. The electrode system possesses typical point-plane geometry, and the needle electrode is made of stainless steel with tip curvature radius of $5 \pm 1 \mu \mathrm{m}$. The ground electrode is $3 \mathrm{~mm}$ away from the needle tip. To keep good contact between needle electrode and XLPE material, the needle electrode was coated with a very thin layer of polyethylene before inserted into the sample. The pretreated needle electrode was inserted into the sample while it was heated in a special mould. For translucent XLPE, the surface smoothness of the sample plays a very important role in obtaining high quality photos.

\section{Experimental set-up and test method}

Figure 1 shows the experimental setup for electrical treeing test. The main equipment is a frequency variable high voltage generator, which consists of a function generator and a frequency variable high voltage amplifier (the amplifier gain is 2000, maximum frequency is $3.5 \mathrm{kHz}$, and the peak-peak value of maximum output voltage is $20 \mathrm{kV}$ ), a live microscope digital camera and a computer system. The samples were immersed in silicone oil during the experiments to enhance the surface insulation and reduce the backlights dispersion from the uneven surface of the sample.

\section{EXPERIMENTAL RESULTS}

A typical experiment was carried out as the follows: (i) setting the sample and testing system as shown in Figure 1; (ii) adjusting the applied voltage frequency to a desirable value on the function generator; (iii) raising the applied voltage to a peak value of $10 \mathrm{kV}$; and (iv) continuously recording the growth

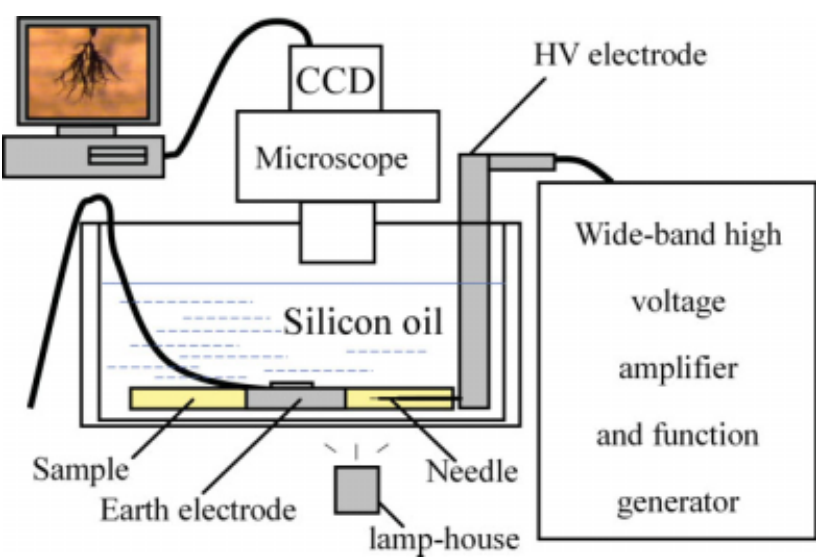

Figure 1 Sample and microscope digital camera system. [Color figure can be viewed in the online issue, which is available at www.interscience.wiley.com.]

characteristics of electrical tree. The recorded data includes initiation time, growth time, and images of electrical trees. Following the above experimental procedure, as many as 50 electrical trees are generated and over 400 images are taken. All the results show a good reproducibility. In this article, the fractal dimension of all electrical trees was estimated by a 2D projected pattern, the fractal dimension was computed by so called box-counting method. ${ }^{8}$

In this article, according to the experimental results, the conducting property of electrical trees can be decided indirectly. When the light-color electrical trees reach the ground electrode, the electrical breakdown does not happen immediately. The resistance between the electrodes measured by a $1000 \mathrm{VM} \Omega$ multi-meter is $>2000 \mathrm{M} \Omega$. On the other hand, once dark-color electrical trees approach the ground electrode the breakdowns always happen quickly. So, it seems that the deposition of carbon can drive the high electrical field to the tips of electrical trees in the case of dark-colored trees.

\section{Electrical trees in XLPE cable samples at low frequency}

At low frequency $(<500 \mathrm{~Hz})$, three types of electrical trees will appear, which are classified by fractal dimension $\left(D_{t}\right)$ : when $D_{t} \leq 1.45$, it is pure sparse branch-like tree [Fig. 2(a)]; when $1.45<D_{t} \leq 1.65$, it is branch-bush mixed tree [Fig. 2(b)]; when $D_{t}>$ 1.65, it is bush-like trees [Fig. 2(c)]. It is clear that the growth rate of the three types is different, pure sparse branch-like tree is the fastest, and the bush tree is the slowest, which demonstrates that the conducting properties differ greatly from one type to another. As to the color of the electrical tree, the pure sparse branch-like tree is the lightest, and the bush tree is the darkest (as shown in Fig. 3), which represent different erosion states (carbon deposition) 

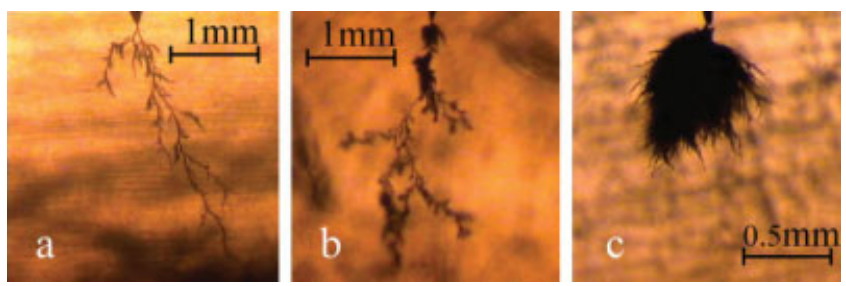

Figure 2 The shapes of electrical tree in $50 \mathrm{~Hz}$. (a) Light branch-like tree, initiation time $9 \mathrm{~min}$, growth time $21 \mathrm{~min}$. (b) Dark mixed tree, initiation time $2412 \mathrm{~min}$, growth time 826 min. (c) Dark bush-like tree, initiation time $80 \mathrm{~min}$, growth time $840 \mathrm{~min}$. [Color figure can be viewed in the online issue, which is available at www.interscience. wiley.com.]

and growth mechanisms in the each electrical tree path. From the photos in Figure 4, at the beginning, the light-color electrical tree grows rapidly [same as Fig. 2(a)], then slows down and its color become dark. It is a typical process that non-conducting trees change into conducting ones.

\section{Electrical trees in XLPE samples at high frequency}

Under high frequency voltage $(f>500 \mathrm{~Hz})$ only dense branch-like trees appear with a fractal dimension between 1.45 and 1.65, as shown in Figure 5. In Figure 5, the fractal dimension of the electrical tree is $1.52,1.46$, and 1.60 , respectively. Generally speaking, the higher the applied frequency, the denser and faster electrical trees with same structure will grow (as shown in Fig. 6). On the other hand, as to the color of the electrical trees, the initiation time of light-color trees is much less than that of dark trees, but the growth rate of the light-color trees is much faster, which is similar to that at low frequency. From the above, it can be concluded that there exist

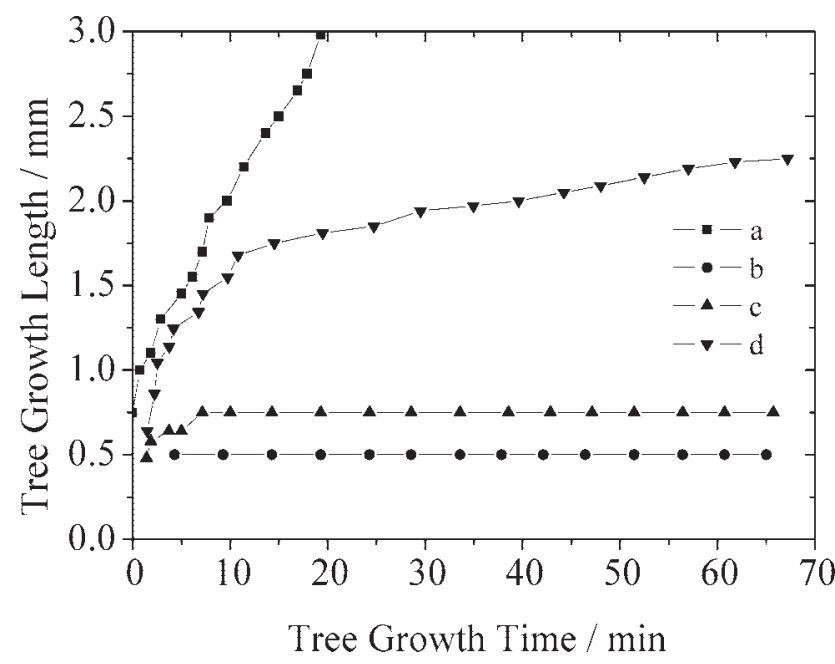

Figure 3 The propagation characteristics of electrical trees under $50 \mathrm{~Hz}$. a, b, c, and d represent the propagation characteristics of a, b, c in Figures 2 and 4, respectively.

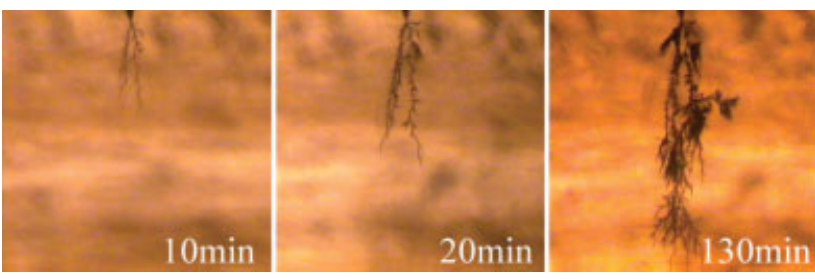

Figure 4 Color changing of an electrical tree in $50 \mathrm{~Hz}$. [Color figure can be viewed in the online issue, which is available at www.interscience.wiley.com.]

two types of conducting structure at high frequency as well, i.e., conducting and non-conducting electrical trees. The growth rate of non-conducting trees is faster than conducting ones.

\section{DISCUSSIONS}

The relationships among initiation time, growth time, color, and fractal dimension of electrical trees with respect to frequency is illustrated in Table I. From the table, it seems that the initiation and growth time of light-color trees are much less than that of dark-color ones, and will be much shorter with the increase of frequency. The fractal dimension of light-color trees will increase at higher frequency. At low frequency, the fractal dimension of light-color tree is less than that of dark-color ones. With the increase of frequency, the initiation time of dark-color will decrease, but frequency has no influence on growth time.

To study the influence of frequency on dark-color trees, a $1 \mathrm{kHz}$ voltage (a peak value of $10 \mathrm{kV}$ ) was applied to the sample as shown in Figure 2(c) for additional $600 \mathrm{~min}$. The results show that the structure and length of dark-color trees changed little, which demonstrates that frequency has no significant effects on dark-color trees. The dark color of electrical tree represents deposition of carbon in paths. So, the conductivity of dark-color trees is larger than that of light-color ones. As the conducting materials in dark-color paths can shift the high electrical field to tips of electrical trees, the partial
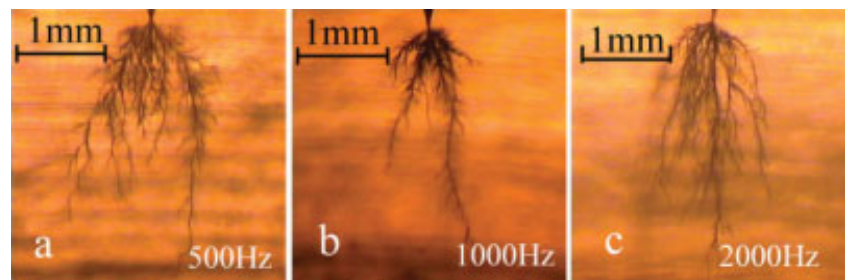

Figure 5 The shapes of electrical trees at high frequency. (a) Initiation time $25 \mathrm{~s}$, growth time $39 \mathrm{~min}$; (b) Initiation time $4 \mathrm{~min}$, growth time $145 \mathrm{~min}$; (c) Initiation time $5 \mathrm{~s}$, growth time $54 \mathrm{~min}$. [Color figure can be viewed in the online issue, which is available at www.interscience. wiley.com.] 


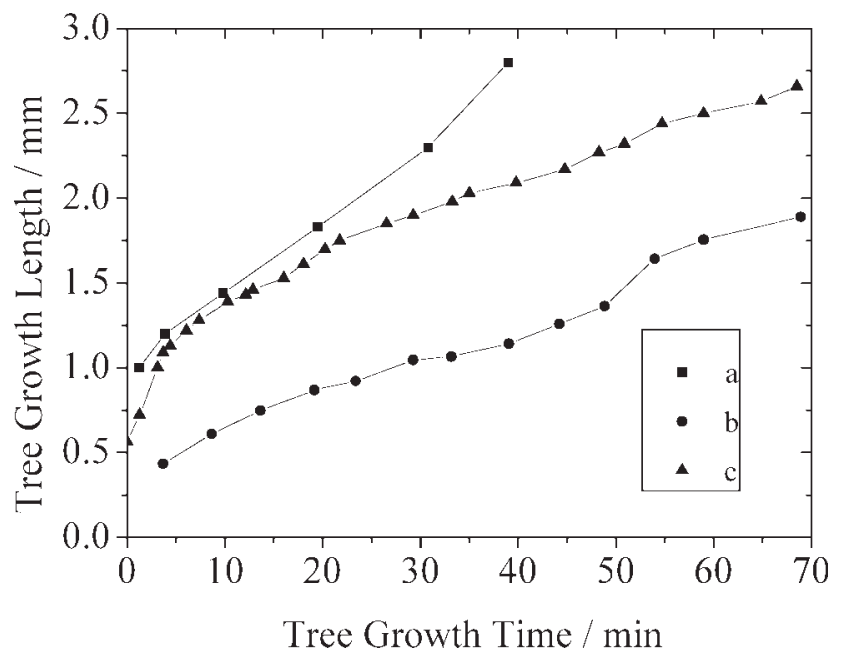

Figure 6 The propagation characteristics of electrical trees at high frequency.

discharge and charge recombination in paths will be less than light-color trees. In view of the strong dependence of partial discharge and high-energy electron recombination on the insulation characteristics of tree paths and the applied frequency, it can be concluded that light-color trees are non-conducting trees, while the dark-color trees are conducting. Furthermore, partial discharge and charge recombination are the main reasons for the fast growth of non-conducting trees; while tiny breakdowns in the tips of electrical trees are responsible for the growth of conducting trees. On the other hand, there are two reasons accounting for the slow propagation of conducting trees: one is the electrical field screening effect among the tips of electrical trees; the other is the screening effect of space charge in material. From the partial discharge measurement of bush-like and branch-like trees in epoxy resin and XLPE, Champion et al. ${ }^{9}$ and Densley et al. ${ }^{10}$ found that under the same condition, the amount of partial discharge in branch-like trees is more than that of bush-like trees, which indirectly indicates that colors can represent different conduction state.

The decrease in the initiation time of electrical trees at high frequency is caused by charge injection and extraction processes from the needle to material, discharge, etc., which will be further enhanced with frequency. ${ }^{11}$

\section{The growth mechanism of non-conducting trees}

The influence of crystallization state on electrical trees has drawn much attention. ${ }^{9,12}$ It is well known that, due to the density difference between crystal and amorphous region, the segregation effect during crystallization and interface effect between rigid crystal structure and flexible amorphous, there will be many charges trapped in boundaries, which will lead to local electrical weak region even micro-pore resulting in a local lower electrical strength. ${ }^{13,14}$ Under the combined influence of ionic impaction

TABLE I

Relationships Among Frequencies of Applying Voltage and Colors, Initiation and Propagation Time, and Fractal Dimension of Electrical Trees

\begin{tabular}{|c|c|c|c|c|c|c|}
\hline \multirow{2}{*}{$\frac{\text { Frequency }}{50 \mathrm{~Hz}}$} & \multirow{2}{*}{$\frac{\text { Parameters }}{\underbrace{(\mathrm{min})}_{\text {Initiation time }}}$} & \multirow{2}{*}{$\begin{array}{c}\begin{array}{c}\text { Light-color } \\
\text { trees }\end{array} \\
9\end{array}$} & \multirow{2}{*}{$\begin{array}{c}\begin{array}{c}\text { Light to dark } \\
\text { trees }\end{array} \\
2 \mathrm{~s}\end{array}$} & \multicolumn{3}{|c|}{$\begin{array}{l}\text { Dark-color } \\
\text { trees }\end{array}$} \\
\hline & & & & 2412 & 80 & \\
\hline & $\begin{array}{l}\text { Growth time } \\
\text { (min) }\end{array}$ & 21 & 130 & 826 & 840 & \\
\hline & $\begin{array}{l}\text { Fractal } \\
\quad \text { dimension }\left(D_{t}\right)\end{array}$ & 1.45 & 1.64 & 1.59 & 1.86 & \\
\hline \multirow[t]{3}{*}{$500 \mathrm{~Hz}$} & $\begin{array}{l}\text { Initiation time } \\
\text { (min) }\end{array}$ & $25 \mathrm{~s}$ & & & & \\
\hline & $\begin{array}{l}\text { Growth time } \\
\text { (min) }\end{array}$ & 39 & & & & \\
\hline & $\begin{array}{l}\text { Fractal } \\
\quad \text { dimension }\left(D_{t}\right)\end{array}$ & 1.52 & & & & \\
\hline \multirow[t]{3}{*}{$1000 \mathrm{~Hz}$} & $\begin{array}{l}\text { Initiation time } \\
\text { (min) }\end{array}$ & $20 \mathrm{~s}$ & & 4 & 16 & 30 \\
\hline & $\begin{array}{l}\text { Growth time } \\
\text { (min) }\end{array}$ & 70 & & 145 & 945 & 410 \\
\hline & $\begin{array}{l}\text { Fractal } \\
\quad \text { dimension }\left(D_{t}\right)\end{array}$ & 1.54 & & 1.46 & 1.63 & 1.59 \\
\hline \multirow[t]{3}{*}{$2000 \mathrm{~Hz}$} & $\begin{array}{l}\text { Initiation time } \\
\quad(\mathrm{min})\end{array}$ & $5 \mathrm{~s}$ & & & & \\
\hline & $\begin{array}{l}\text { Growth time } \\
\text { (min) }\end{array}$ & 54 & & & & \\
\hline & $\begin{array}{l}\text { Fractal } \\
\quad \text { dimension }\left(D_{t}\right)\end{array}$ & 1.60 & & & & \\
\hline
\end{tabular}


effect caused by PD, melting effect caused by local high-temperature, expansion effect caused by local high-pressure, and the recombination effect of high energy electrons in non-conducting tree path, the non-conducting trees along boundaries of big spherulites will grow very fast [Figs. 2(a) and $5(\mathrm{a}, \mathrm{c})]$.

The influences of partial discharge on non-conducting trees can be described as follows: the model for electrical tree path is assumed as shown in Figure 7 , and then the series capacity is

$$
C_{b}=\frac{\varepsilon_{r} \varepsilon_{0} A}{3-L}
$$

where $L$ is the length of single tree $(\mathrm{mm}), A$ is the cross area of tree path, $\varepsilon_{r}$ is the relative permittivity of XLPE. Assume that the breakdown field of the gas in tree paths is $E_{b}$, and then the change of voltage across the tree can be approximately expressed as $\Delta U=E_{b} L$, so the amount of one discharge is $Q=$ $C_{b} \Delta U$, put the above into (1),

$$
Q=\varepsilon_{r} \varepsilon_{0} A E_{b} \frac{L}{3-L}
$$

and then differentiate (2)

$$
\frac{d L}{d t}=K(3-L)^{2} \frac{d Q}{d t}
$$

where $K=1 /\left(3 \varepsilon_{r} \varepsilon_{0} A E_{b}\right)$. According to eq. (3), the growth rate of non-conducting trees is proportional to the change rate of discharge amount, which is consistent with the fast growth characteristic of light-color branch-like tree at high frequency.

\section{The growth mechanism of conducting trees}

When the morphology of polymer is uniformly distributed small spherulites, or the trees grow into the big spherulites at the beginning, dark-color conducting trees or conducting/non-conducting mixed trees will form, just as shown in Figures 2(b,c), 4, and 8. Figure 8(a) represents the shape of the initiating tree,

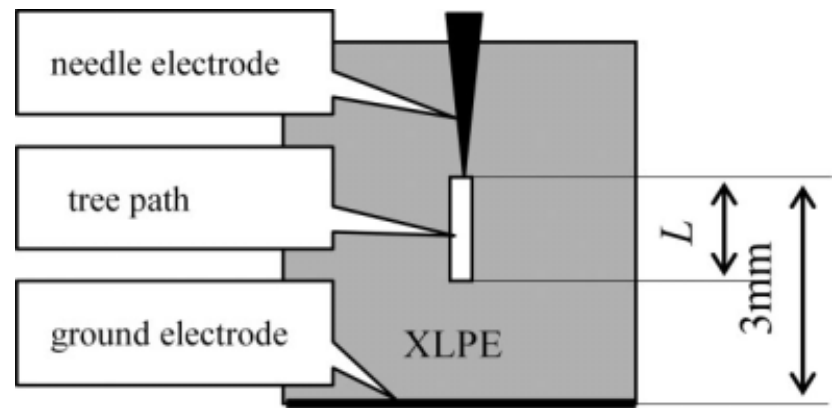

Figure 7 The model of non-conducting electrical tree with single path.

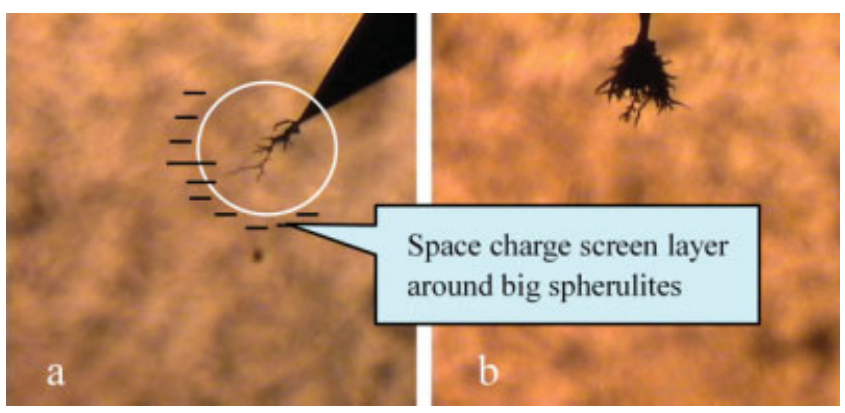

Figure 8 A conducting electrical tree that nearly stopped propagation and model of space charge screen layer. (a) Initiation time $80 \mathrm{~min}$ at $500 \mathrm{~Hz}$; (b) Growth time $845 \mathrm{~min}$ at $50 \mathrm{~Hz}$. [Color figure can be viewed in the online issue, which is available at www.interscience.wiley.com.]

Figure $8(\mathrm{~b})$ shows the situation at $845 \mathrm{~min}$, after which the frequency was changed to $1 \mathrm{kHz}$ while the applied voltage was kept the same for an additional $640 \mathrm{~min}$. The additional time has no significant effect on the length and structure of the tree, which indicates that the model shown in Figure 7 and eq. (3) can not be used to explain the growth characteristics shown in Figures 2(b,c), 4, and 8. The conduction characteristics shown here can be interpreted as below.

As shown in Figure 8, during the initiation period, the tree is black and grows slowly, so the conductivity of the electrical tree path is large and partial discharge seldom happens. The screening effect caused by space charges injected from the tips of trees into amorphous regions around spherulites will suppress the local high field in the tips of trees. The screening effect can be approximately estimated as follows.

On the basis of the enlarged photos of electrical trees, the curvature radius of tips of trees should be much smaller than that of stainless steel needle (5 $\mu \mathrm{m})$. Assuming that the length of tree is $0.5 \mathrm{~mm}$, thus the remaining distance between two electrodes is $2.5 \mathrm{~mm}$, and the sample is free of space charges and the curvature radius of tips of trees is $5 \mu \mathrm{m}$, then the minimum field at the tips of the trees is:

$$
E_{\min }=\frac{U}{r_{1}-\frac{r_{1}^{2}}{r_{2}}}=\frac{7 \times 10^{3}}{0.5 \times 10^{-5}-\frac{\left(0.5 \times 10^{-5}\right)^{2}}{2.5}} \approx 1400 \mathrm{MV} / \mathrm{m}
$$

but the breakdown electric strength of $\mathrm{PE}$ is $18-28$ $\mathrm{MV} / \mathrm{m}^{6}$ i.e., the actual electric field is $50-80$ times higher than the breakdown strength of PE, which would of course lead to a fast grow of the electrical trees. However, the electrical trees grow very slowly. This fact suggests that in this case there must exist a smooth screening layer of space charge [Fig. 8(a)], which will reduce the local electrical field of space charge layer to some value less than $E_{b}$. Thus, assuming the space charge layer is a semi-sphere 
layer with a radius of $r_{s}$ [Fig. 8(a)], the minimum size of the layer can be estimated as follows:

$$
E_{b}=\frac{U}{r_{s}-\frac{r_{s}^{2}}{r_{2}}}=\frac{7 \times 10^{3}}{r_{s} \times 10^{-6}-\frac{\left(r_{s} \times 10^{-6}\right)^{2}}{2.2}}=28 \mathrm{MV} / \mathrm{m}
$$

This leads to a radius $r_{s} \approx 250 \mu \mathrm{m}$, which is the possible size of spherulites in XLPE. ${ }^{13}$ Considering the density difference of crystal and amophous region, defects (electron traps), micro-pore distribution along spherulites boundaries, and the relationship between space charge and aging of electrical tree in insulation, ${ }^{9,15}$ especially the energy band model proposed by Tanaka ${ }^{11}$ regarding the injection of electron-type space charge into PE and captured by traps in weak region, it is suggested that if the electrical tree grow into the spherulite, it can form a layer of space charge along the boundaries of spherulites, thus inhibiting the growth of conducting trees.

\section{CONCLUSIONS}

1. The conduction characteristics of electrical trees in XLPE depend on the morphology of the material. The electrical tree paths in big spherulites or among uniform small spherulites are conducting path, while the electrical trees in amorphous regions along the boundaries are nonconducting.

2. The space charge limited tiny breakdown at the tips of electrical trees is responsible for the propagation process of conducting trees. Because of the screen effect of space charges around tips of electrical trees or at spherulites boundaries, the conducting trees grow slower than non-conducting ones. When electrical trees grow into spherulites or uniform small spherulites, the growth rate will decrease greatly.

3. Increasing the crystallinity and decreasing the size of spherulites are the key points in the material itself to inhibit electrical trees.

\section{References}

1. Kaneiwa, H.; Suzuoki, Y.; Mizutani, T. IEEE Trans Dielectr Electr Insul 2000, DEI-7, 843.

2. Kaneiwa, H.; Suzuoki, Y.; Mizutani, T. IEEE Trans Dielectr Electr Insul 2001, DEI-8, 72.

3. Rainer, P.; Martin, H. IEEE International Conference on Conduction and Breakdown in Solid Dielectrics; U-25: Sweden, 1998.

4. Sarathi, R.; Supriyo, D.; Anil Kumar, C. R.; Velmurugan, R. J Appl Polym Sci 2004, 92, 2169.

5. Li, Z.; Yin, Y.; Wang, X.; Tu, D. M.; Kao, K. C. J Appl Polym Sci 2003, 89, 3416.

6. Champion, J. V.; Dodd, S. J. J Phys D: Appl Phys 2001, 34, 1235.

7. Champion, J. V.; Dodd, S. J.; Zhao, Y. IEEE Trans Dielectr Electr Insul 2001, DEI-8, 284.

8. Kudo, K. IEEE Trans Dielectr Electr Insul 1998, DEI-5, 713.

9. Dissado, L. A. IEEE Trans Dielectr Electr Insul 2002, DEI-9, 483.

10. Densley, J.; Kalicki, T.; Nodolny, Z. IEEE Trans Dielectr Electr Insul 2001, DEI-8, 48.

11. Tanaka, T. IEEE Trans Dielectr Electr Insul 2001, DEI-8, 733

12. Zhao, Y.; Vaughan, A. S.; Champion, J. V.; Dodd, S. J.; Sutton, S. J. Dielectr Mater Meas Appl Conf IEEE 2000, 473, 314.

13. Wu, S. Z.; Xie, D. R.; Chen, S. T.; Yu, B. L. Electrical Insulation Material Science and Engineering; $\mathrm{Xi}^{\prime}$ an Jiaotong University press: Xi'an, 1996.

14. Liu, Z. Y.; Wang, H. M. The Design Principal of Power Cable Structure; Xi'an Jiaotong University press: Xi'an, 1996.

15. Dang, Z. M.; Kang, J.; Tu, D. M. Proc CSEE 2001, 21, 5. 